\title{
Neuere Entwicklungen der X-PAP-Therapie für obstruktive und zentrale schlafbezogene Atmungsstörungen
}

\author{
New Developments in X-PAP Therapy for Obstructive and Central \\ Sleep-Disordered Breathing
}

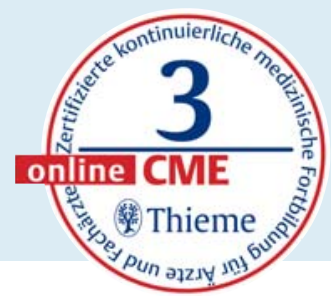

K.-H. Rühle ${ }^{1,2}$, K. J. Franke ${ }^{1,2}$, U. Domanski ${ }^{1,2}$, M. Schröder ${ }^{1,2}$, G. Nilius ${ }^{1,2}$

${ }^{1}$ Klinik für Pneumologie, HELIOS-Klinik Ambrock Hagen

2 Universität Witten-Herdecke, Lehrstuhl Innere Medizin I

\section{Lernziele}

$\nabla$

Aufgrund der hohen Prävalenz von schlafbezogenen Atmungsstörungen, insbesondere der obstruktiven Schlafapnoe, wird die Pneumologie häufig mit apparativen Therapieproblemen konfrontiert. In dem vorliegenden Artikel werden sowohl die Standardtherapie mit kontinuierlich positivem Überdruck (CPAP) als auch die verschiedenen Modifikationen mit positivem Atemwegsdruck, wie zum Beispiel automatisch positiver Atemwegsdruck (APAP), Beatmung mit zwei Druckniveaus (BPAP) sowie Druckentlastungsverfahren (pressure relief) vorgestellt. Lernziel sollte es sein, die Funktionsweise der verschiedenen apparativen Methoden kennen zu lernen, um ein tieferes Verständnis für die einzelnen Indikationen zu entwickeln.

\section{Einleitung}

Die Therapietreue von Patienten mit schlafbezogenen Atmungsstörungen (SBAS), die mit kontinuierlich positivem Atemwegsdruck (CPAP) behandelt werden, ist in vielen Fällen nicht optimal. Die Akzeptanz bzw. Adhärenz einer Beatmungstherapie von Patienten mit obstruktiver Schlafapnoe (OSA) liegt nach neueren Studien bei etwa 75\% der Patienten, bei denen eine CPAP-Therapie indiziert ist. So fand eine deutsche Arbeitsgruppe innerhalb der ersten drei Monate nach Therapiebeginn eine Abbruchquote von etwa $10 \%$. Bei einer Kontrolle nach etwa einem Jahr nutzten mehr als 25\% aller Patienten die Therapie nicht mehr [1]. Da eine klare Korrelation zwischen Anwendungsdauer und Effektivität bzgl. Tagesschläfrigkeit besteht, wird eine ausreichend lange Anwendungsdauer pro Nacht angestrebt. Als Maß für eine ausreichende Adhärenz wird heute häufig ein Schwellenwert von 4 Stunden angenommen. Jedoch sollte die Therapiedauer pro Nacht aufgrund großer individueller Unterschiede in Abhängigkeit vom klinischen Ansprechen festgelegt werden. Eine Mindesttherapiedauer von 4 Stunden/Nacht ist nicht in jedem Fall erforderlich [2]. Die Adhärenz hängt von vielen Faktoren $a b$, unter anderem von der Beatmungsqualität, aber natürlich auch von der inneren Einstellung des Patienten. Eine ausreichende Adhärenz ist zu erwarten, wenn zum Beispiel nach der ersten Titrationsnacht mit CPAP keine relevanten Probleme berichtet werden [3].

Unter anderem wird der Druck, gegen den die Patienten atmen müssen, als unangenehm bzw. sehr gewöhnungsbedürftig angesehen. Deshalb wurden verschiedene Modifikationen der Druckanwendung entwickelt, um die Beatmungstherapie angenehmer zu gestalten (X in X-PAP steht für die verschiedenen Druckmodifikationen). Die Modifikationen betreffen sowohl den Charakter des Druckverlaufs während der gesamten Nacht als auch die Druckanwendung während eines einzelnen Atemzugs. Im Folgenden werden insbesondere die neueren Entwicklungen auf dem Gebiet der apparativen positiven Druckanwendung (PAP) mit ihren Vorteilen und Einschränkungen dargestellt ( Tab.1).

Tab. 1 Übersicht zu den verschiedenen apparativen Techniken zur Behandlung von Patienten mit schlafbezogenen Atmungsstörungen.

Verfahren mit fixem Druck
- CPAP (Kontinuierlich positiver Atemwegsdruck)
- OPAP (Offener positiver Atemwegsdruck)
Verfahren mit variablem Druck
- APAP ( Automatisch positiver Atemwegsdruck)
- AWPAP (Schnelle Druckreduktion im Wachzustand,
SensAwake ${ }^{\circledR}$ )
Verfahren mit Druckreduktion während Exspiration
- BPAP (Bilevel positiver Atemwegsdruck)
- C-Flex ${ }^{\circledR}$ bei CPAP und APAP (Pressure Relief)
- Bi-Flex ${ }^{\circledR}$ bei BPAP
Verfahren mit inspiratorisch erhöhtem und
exspiratorisch erniedrigtem Atemwegsdruck
- Flexline ${ }^{\circledR}$, A-Flex ${ }^{\circledR}$
Verfahren mit exspiratorisch erhöhtem positiven
Atemwegsdruck
- EPAP (Exspiratorisch positiver Atemwegsdruck)
Adaptive Verfahren
- SPAP (Adaptive Servoventilation, ASV)

VNR

2760512013141214321

\section{Bibliografie}

DOI http://dx.doi.org/

10.1055/s-0033-1344494

Pneumologie 2013; 67: 551-561

(c) Georg Thieme Verlag KG

Stuttgart · New York

ISSN 0934-8387

Korrespondenzadresse

Prof. Dr. med. Karl-Heinz Rühle

HELIOS-Klinik Ambrock

Klinik für Pneumologie

Ambrockerweg 60

58091 Hagen

Klinik-Ambrock.Pneumo@

t-online.de 


\section{Verfahren mit fixem Druck} $\nabla$

\section{CPAP (Kontinuierlich positiver Atemwegs- druck)}

Die Behandlung der mittelgradigen und schwergradigen obstruktiven Schlafapnoe mit kontinuierlich positivem Atemwegsdruck hat sich seit vielen Jahren als Standardtherapie herauskristallisiert [4]. Seit einigen Jahren existieren in Europa technische Mindestkriterien, die durch die Geräte erfüllt werden sollten. Nur die Verordnung normgerechter Geräte mit normgerechter Gebrauchsanweisung ist zulässig (siehe $\bullet$ Tab. 2). Nach Indikationsstellung mittels Polygrafie bzw. Polysomnografie wird durch eine nächtliche Titration der Behandlungsdruck gefunden, der sowohl die Apnoen als auch die Hypopnoen, Schnarchen, $\mathrm{O}_{2}{ }^{-}$ Desaturation und respiratorisch bedingte Weckreaktionen (RERAs) verhindert. Bei schlechter Toleranz bzw. Intoleranz des Beatmungsdrucks können verschiedene Modifikationen des CPAPVerfahrens zum Einsatz kommen.

\section{OPAP (Offener positiver Atemwegsdruck)}

Da manche Patienten die Maske oder den zuführenden Schlauch nicht tolerieren, wurde ein Verfahren entwickelt, das lediglich eine Nasenbrille oder Nasenkanüle verwendet. Die Luft wird mit hoher Flussrate über die beiden Nasenöffnungen (nasal High Flow, nHF) zugeführt, ohne dass eine Maske erforderlich wäre. Mit dieser so genannten transnasalen Insufflation wird supraglottisch bei einer Flussrate von 301/min ein Druck von etwa 2 und bei $40 \mathrm{l} / \mathrm{min}$ etwa $3 \mathrm{~cm} \mathrm{H}_{2} \mathrm{O}$ gemessen [5]. In einer ersten Untersuchung wurden Patienten mit allen Schweregraden von OSA mit der transnasalen Insufflation über eine offene Nasenkanüle therapiert [6]. Es kam zu einer deutlichen Reduktion des Apnoe/Hypopnoe-Index. Vor allem Patienten mit Hypopnoen profitierten am meisten. Da der Schlauch einen sehr geringen Durchmesser von wenigen Millimetern aufweist, muss der treibende Druck erhöht werden, um den hohen Widerstand in der Zuführung zu überwinden. Mit dem Kompressor des Gerätes wird ein Druck von mehr als 250 mbar erzeugt. Durch die hohe Flussrate würden die Nasenschleimhäute sehr schnell auskühlen und austrocknen. Dies wird dadurch verhindert, dass die Atemluft durch eine Schlauchheizung auf $30-32{ }^{\circ} \mathrm{C}$ angewärmt und die Luftfeuchtigkeit auf über $80 \%$ erhöht wird. Neben einem kompressorbetriebenen Gerät TNI $^{\circledast}$ (Firma TNI Medical) ist ein weiteres Gerät zur transnasalen Insufflation auf dem Markt, das den hohen Fluss mit einer Turbine generiert (Air$v^{\circledR}{ }^{\circledR}$, Firma Fisher und Paykel). In einer größeren Untersuchung an 56 Patienten konnte durch transnasale Insufflation der AHI reduziert werden. Auch hier bestätigte sich, dass die höchste Erfolgsrate bei Patienten mit überwiegend $\mathrm{Hy}$ popnoen zu verzeichnen war. Lag die prozentuale Hypopnoerate während der Basisuntersuchung
Tab. 2 Zusammenstellung der Daten, die nach DIN EN 17510-1: 2009-07 in der Gebrauchsanweisung von PAPGeräten gefordert wird.

Stabilität des statischen Drucks (Langzeitgenauigkeit)
Stabilität des dynamischen Drucks
- Bei verschiedenen Prüfdrucken und
- verschiedenen Atemfrequenzen
Stabilität des dynamischen Drucks unter Berücksich-
tigung klinischer Kriterien bei + $/ 0,3 \mathrm{hPa}$.
Angabe der maximalen Durchflussrate
Angabe des maximalen Schalldruckpegels

bei über $90 \%$, war die transnasale Insufflation bei $50 \%$ der Patienten erfolgreich, d.h. der AHI fiel unter 10/h [7]. Bei überwiegend obstruktiven Apnoen lag die Erfolgsrate dagegen bei lediglich 18\% aller Patienten. Zusammenfassend zeichnet sich ab, dass das offene CPAP-Verfahren bei Untergruppen wie zum Beispiel Erwachsenen mit überwiegend Hypopnoen und Patienten mit Schnarchen indiziert sein kann. Die transnasale Insufflation könnte damit eventuell als Alternative zu CPAP bei geringen Schlaf-Atmungsstörungen infrage kommen.

Die größte Erfolgsrate unter nasaler High-Flow-Therapie ist bei Atmungsstörungen mit überwiegend Hypopnoen zu erwarten.

\section{Verfahren mit variablem Druck}

$\nabla$

APAP (Automatisch positiver Atemwegs-

druck)

Die automatischen Beatmungsgeräte verändern den Beatmungsdruck zur Beseitigung der Obstruktion in Abhängigkeit vom jeweiligen Obstruktionsgrad der oberen Atemwege. Somit gelingt es, den jeweiligen Druck an die variable Obstruktion anzupassen. Zur Steuerung des Druckverhaltens werden verschiedene Atmungssignale verwendet. Das am häufigsten eingesetzte Verfahren beinhaltet den Atemfluss (Flow) mit genauer Analyse von Amplitude und Abflachung des Flusssignals (Flattening). Zusätzlich werden schnelle Druckschwankungen infolge des Schnarchens zur Steuerung verwendet.

Mittels forcierter Oszillations-Technik (FOT) wird dem Atemstrom kontinuierlich ein oszillierender Druck aufgeprägt. Aus den resultierenden Druckschwankungen kann der Widerstand in den Atemwegen gemessen werden.

Mit einem weiteren Verfahren kann zwischen zentralen und obstruktiven Ereignissen differenziert werden. Jeweils zum Ende einer obstruktiven Phase entsteht bei Wiedereröffnung der Atemwege ein kurzer Unterdruck, der mit einem sensiblen Drucksensor erfasst werden kann (obstructive pressure peak, OPP) [8]. Handelt es sich um eine Apnoe ohne Verschluss der Atemwege, fehlt dieses Signal. 
In einer Vergleichsstudie zwischen APAP und CPAP konnte eine über die Nacht integrierte Druckerniedrigung um 2 mbar zu Gunsten von APAP erzielt werden [9]. Aufgrund der Regelungscharakteristik von APAP lag der maximale Druck im Vergleich zu CPAP um etwa 4 mbar höher. Die Schlafqualität war allerdings nicht verbessert. Nach Befragung der Patienten nach der Präferenz bevorzugten drei Viertel der Patienten das automatisch regulierende Gerät.

Der Einsatz von APAP hat sich bei einigen Untergruppen von OSA-Patienten als sinnvoll herausgestellt. Vor allem bei Patienten mit höherem Druckbedarf konnte durch Verwendung eines APAP-Gerätes eine signifikant höhere Nutzungszeit nachgewiesen werden [10]. Patienten mit ausgeprägter Abhängigkeit der Atmungsstörung von der Körperposition oder den Schlafstadien, hier insbesondere den REM-Phasen, profitieren ebenfalls von der automatischen Druckanpassung, da der Druck während der überwiegenden Zeit der Nacht abgesenkt werden kann [11]. In einer Cochrane-Analyse, bei der APAP mit CPAP verglichen wurde, konnten 30 Studien mit über 1100 Patienten ausgewertet werden [12]. Zwar fand sich eine signifikante Differenz der Nutzungszeiten von 0,21 Stunden. Dabei ist zu hinterfragen, ob es sich tatsächlich um eine klinisch relevante Verlängerung der Nutzungszeit handelt. In den meisten Studien fand sich eine Präferenz der Patienten für die APAP-Therapie.

Die Adhärenz von Patienten mit hohem Druckbedarf unter CPAP kann mit automatisch positivem Atemwegsdruck (APAP) verbessert werden.

\section{AWPAP (Schnelle Druckreduktion im Wachzustand, SensAwake ${ }^{\circledR}$ )}

Viele Patienten empfinden den Druck nach dem Aufwachen mit mehrere Minuten dauernden Wachphasen als relativ unangenehm. Ein hoher positiver Atemwegsdruck während der Wachphasen ist allerdings nicht erforderlich, da während dieser Zeit keine obstruktiven Ereignisse auftreten. Es konnte nun gezeigt werden, dass in der Wachphase unregelmäßige Atemflussmuster (Amplituden- und Frequenzschwankungen) auftreten, die visuell ausgewertet, aber auch durch einen entsprechenden Algorithmus identifiziert werden können [13]. Die mit einem neuronalen Netzwerk gefundenen Algorithmen zur Erfassung der Wachphasen wurden in einem APAP-Gerät implementiert (Sensawake ${ }^{\circledR}$ ) und konnten zur automatischen schnellen Druckabsenkung während Wachphasen genutzt werden [14] ( $\bullet$ Abb. 1)

Die Detektion von Wachphasen mit unregelmäßigem Atemmuster kann mit geeigneten Algorithmen erkannt und zur Einleitung einer schnellen Druckabsenkung unter APAP-Therapie verwandt werden.

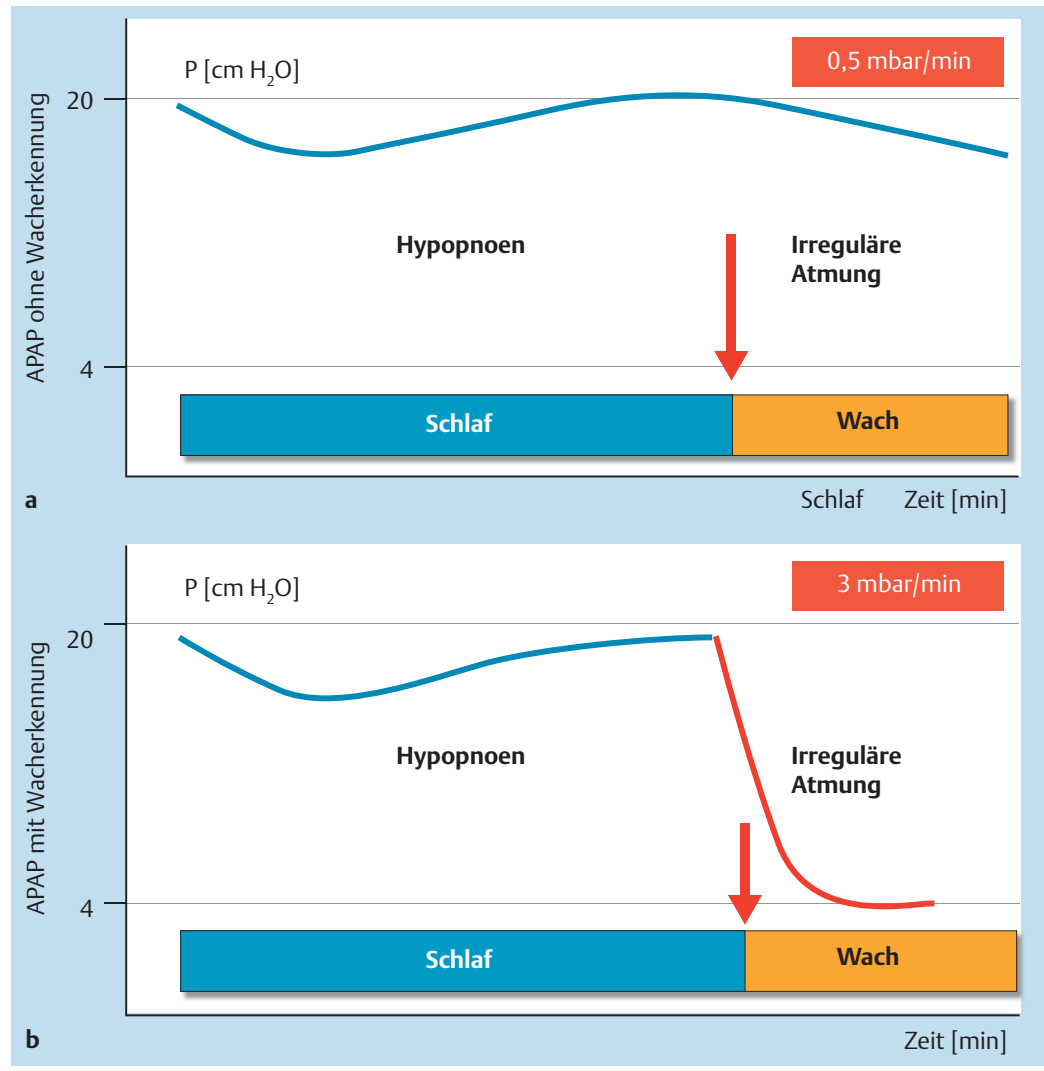

Abb. 1 Schema von APAP a ohne Wacherkennung mit langsamer Druckabsenkung (0,5 mbar /min) im Wachzustand, da weder Apnoen noch Hypopnoen während der Wachphase auftreten. b APAP mit Wacherkennung mit schneller Druckabsenkung bei Wachphasen (Sensawake) ${ }^{\circledR}$ durch Erkennung irregulärer Atmung.

In einem intraindividuellen Vergleich an $42 \mathrm{~Pa}-$ tienten konnte mit dieser Technologie eine geringe, aber signifikante Druckabsenkung gegenüber APAP gezeigt werden [15]. Allerdings fand sich kein signifikanter Unterschied, wenn die Wachzeiten nach Schlafbeginn (WASO) verglichen wurden. Auch die sonstigen Schlafparameter zur Charakterisierung der Schlafqualität änderten sich unter der schnellen Druckabsenkung während der Wachphasen nicht. Die Studie überprüfte allerdings nur jeweils eine Nacht mit und ohne Druckabsenkung während der Wachphasen.

\section{Verfahren mit Druckreduktion während Exspiration \\ $\checkmark$}

BPAP (Bilevel positiver Atemwegsdruck)

Manche OSA-Patienten, bei denen ein hoher Druckbedarf besteht, sind häufig nicht in der Lage, gegen den hohen Druck auszuatmen. Besteht eine solche Druck-Dyspnoe, wird nach Alternativen gesucht, wobei neben APAP eine Drucktherapie mit zwei unterschiedlichen Druckniveaus infrage kommt. Die Methode gestattet, voneinander unabhängig den Druck während Exspiration (EPAP) und Inspiration (EPAP) anzupassen. Durch den positiven endexspiratorischen Druck kann ein Kollaps der oberen Atemwege während der Exspiration vermieden werden. Ein höherer IPAP

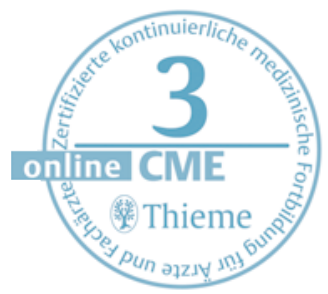




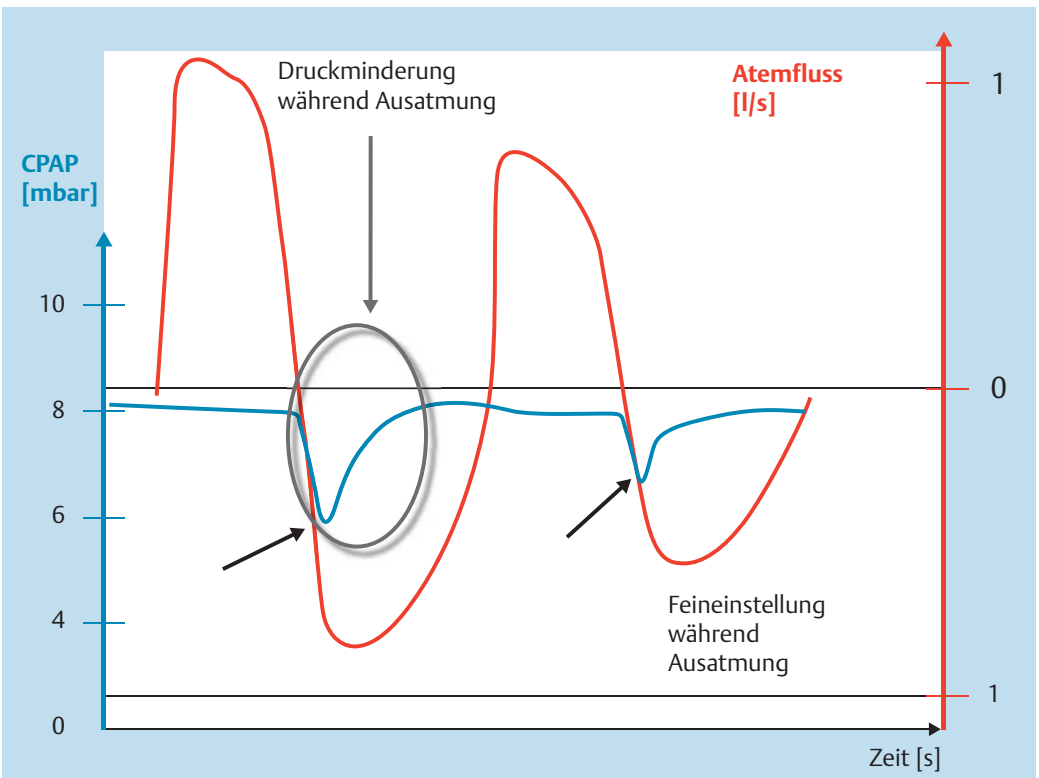

Abb.2 Modifizierte CPAP-Atmung (C-Flex). Während der Esxpiration wird der PAP kurzfristig abgesenkt, steigt aber zu Beginn der Inspiration wieder auf das vorgegebene CPAP-Niveau an. Die Druckentlastung ist von der Höhe des exspiratorischen Flusses abhängig [20].

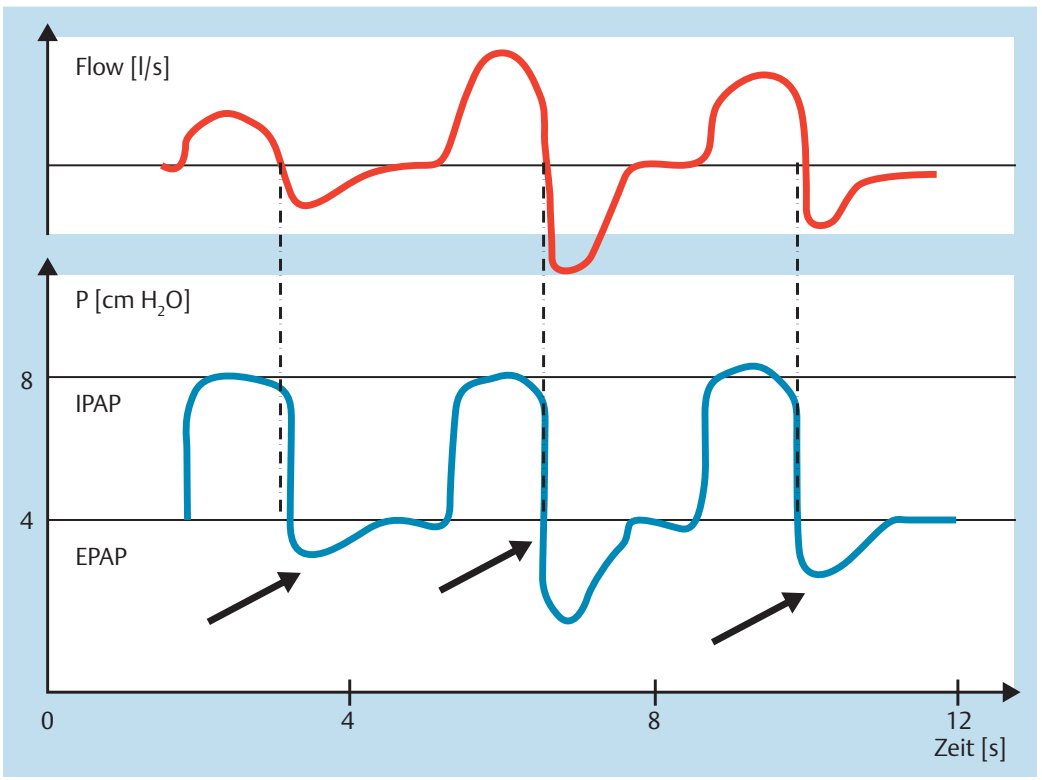

Abb.3 Bilevel mit Druckentlastung. Flow-proportionale Druckabsenkung des EPAP (siehe Pfeile).

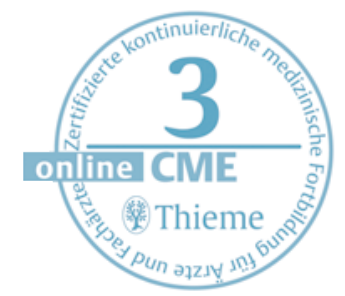

ist erforderlich ist, um die höhere Kollapsneigung während der Inspiration zu verhindern und Hypopnoen mit $\mathrm{O}_{2}$-Desaturation zu vermeiden [16]. In kleineren Vergleichsstudien von BPAP versus CPAP resultierte durch BPAP allerdings kein relevanter Vorteil bezüglich höher Adhärenz oder Verbesserung der schlafbezogenen Atmungsstörung [4]. Allerdings bietet das Verfahren gewisse Vorzüge bei der Behandlung von Untergruppen [17]. Etwa 25\% der Patienten mit Intoleranz gegenüber hohen CPAP-Drücken, die bei persistierenden Hypopnoen mit $\mathrm{O}_{2}$-Desaturation notwendig wurden, konnten auf BPAP umgestellt werden [18]. In einer Analyse bei mehr als 2500 Patienten, die entweder CPAP oder BPAP erhielten, wurden die Erkrankungen der Patienten ermittelt, die primär auf eine BPAP-Therapie eingestellt oder die später von CPAP auf die BPAP-Therapie umgestellt wurden. Die Korrelate für eine BPAP-Therapie waren höheres Alter, Body Maß Index $(\mathrm{BMI})>35$, chronisch obstruktive Lungenerkrankung (COPD), schwergradiges obstruktives Schlafapnoesyndrom, schwergradige $\mathrm{O}_{2}$-Desaturation und Tagesschläfrigkeit [19]. Sollte eine solche Konstellation oder die Zugehörigkeit zu einer dieser Patientengruppen vorliegen, verspricht in diesen Fällen die primäre Verordnung einer BPAP-Therapie im Vergleich zu CPAP die höhere Adhärenz.

Patienten mit OSA und pathologischem Übergewicht und/oder COPD profitieren häufig von einer Ein- oder Umstellung auf eine BPAP-Therapie (Bilevel positiver Atemwegsdruck)

C-Flex ${ }^{\circledR}$ bei CPAP und APAP (Pressure Relief) Hinter dem Druckabsenkungsverfahren zu Beginn der Ausatmung steht die Vorstellung, dass die Atmung unter CPAP dadurch erleichtert und die Akzeptanz einer kontinuierlichen Überdruckatmung erhöht wird. Die Druckabsenkung hängt von der Geschwindigkeit der Ausatmung ab. Bei dem Gerät C-Flex ${ }^{\circledR}$ (Fa Respironics) stehen drei Stufen für die Druckabsenkung zur Verfügung. Bei der höchsten Stufe 3 liegt die maximale Druckabsenkung bei etwa $30 \%$ des gewählten CPAP-Niveaus [20]. Da die Kollapsneigung der oberen Atemwege vor allem zu Beginn der Inspiration am höchsten ist, wird der reduzierte Druck bei dem Verfahren schon vor Beginn der folgenden Inspiration auf das voreingestellte CPAP-Niveau angehoben ( $\bullet$ Abb. 2).

Eine deutliche Druckentlastung findet sich vor allem bei hohen Atemfrequenzen und CPAP-Werten über 8 mbar. Damit unterscheidet sich das Verfahren von der BPAP-Therapie, bei der der Druck erst zu Beginn der Einatmung erhöht wird. In einer Studie an 51 Patienten mit einer CPAPTherapie mit und ohne Flex-Technologie konnte die Äquivalenz beider Therapieformen nachgewiesen werden [21]. Die Adhärenz der untersuchten Patientengruppe lag bei über 5 Stunden pro Nacht, was einer sehr guten Nutzungszeit entspricht. Die kurze Druckreduktion in der Ausatmung dürfte die Nutzungszeit vor allem bei Patienten mit verminderter Adhärenz infolge von Druckproblemen positiv beeinflussen. Häufig wird das Flex-Verfahren in Kombination mit anderen Methoden zur Druckreduktion (z.B. APAP oder BPAP) kombiniert ( $\mathbf{A b b . 3 )}$.

Bei den CPAP-Druckentlastungsverfahren (C-Flex ${ }^{\circledR}$ ) wird der Druck abhängig von der Geschwindigkeit der Ausatmung reduziert, wird aber schon vor Beginn der folgenden Inspiration wieder auf das vorgegebene CPAP-Niveau angehoben 


\section{BPAP mit Pressure Relief}

Der Effekt einer BiLevel-Therapie mit Druckentlastung wurde bei einer Gruppe von 104 Patienten, bei der die Adhärenz unter 4 Stunden pro Nacht lag, überprüft. Bei der einen Hälfte der Patienten wurde CPAP fortgeführt, die andere Hälfte erhielt eine Behandlung mit BPAP und zusätzlicher Druckentlastung. Nach jeweils 3 Monaten wurde die Adhärenz überprüft. In der CPAPGruppe nutzten 28\% die CPAP-Therapie mehr als 4 Stunden pro Nacht, in der mit Bi-Flex behandelten Gruppe fand sich ein signifikant höherer Anteil mit 49\% [22].

\section{Auto-BPAP mit Pressure Relief}

Bei diesem Verfahren werden exspiratorischer (EPAP) und inspiratorischer Druck (IPAP) abhängig von der Obstruktion geregelt. Bei Apnoen und Schnarchen wird der exspiratorische Druck angehoben. Bei obstruktiven Hypopnoen wird der inspiratorische Druck erhöht. Hinzu kommt bei jedem Atemzug die Druckentlastung bei der Exspiration (siehe Abb.4). Eine weitere Optimierung des Druckes wird durch eine regelmäßige Überprüfung der Kontur der Flusskurve angestrebt. Nach 10 Minuten normaler Atmung wird der inspiratorische Druck langsam abgesenkt, bis sich die Zeichen einer Flusslimitation ergeben. Danach folgt in diesem kontinuierlichen, proaktiven Prozess ein langsamer Druckanstieg, bis sich die Flusskontur völlig normalisiert hat und damit eine optimale Druckeinstellung besteht.

In einer Studie an Risikopatienten wurde das Verfahren überprüft [23]. 47 OSA-Patienten mit während der Titrationsperiode geringer Schlafeffizienz von weniger als 70\% und mehr als 20 Arousals pro Stunde wurden als Risikogruppe eingestuft, die Risikogruppe wurde ergänzt von Patienten, die die Titration abgebrochen hatten und die der Arzt als gering compliant einschätzte. Als Erfolgskriterium einer guten Einstellung wurden Nutzungszeiten von mehr als 4 Stunden definiert. Unter Auto-BPAP erreichten 62\%, unter CPAP nur $52 \%$ der Patienten diesen Schwellenwert, die Differenz war allerdings nicht signifikant. Somit zeigte sich tendenziell in einer ausgewählten Risikogruppe eine Besserung unter dem aufwändigeren Druckverfahren.

\section{Verfahren mit inspiratorisch erhöhtem und exspiratorisch erniedrigtem Atem- wegsdruck}

\section{Flexline ${ }^{\circledR}$ und A-Flex ${ }^{\circledR}$}

Eine weitere Modifikation des einzelnen Atemzugs wurde in einem Gerät mit dynamischer Druckcharakteristik mit inspiratorischer Druckanhebung und exspiratorischer Druckabsenkung realisiert (Flexline ${ }^{\circledR}$ ). Dieses Verfahren kann sowohl im CPAP-Modus, aber auch im BPAP- oder APAP-Modus implementiert werden. An zehn

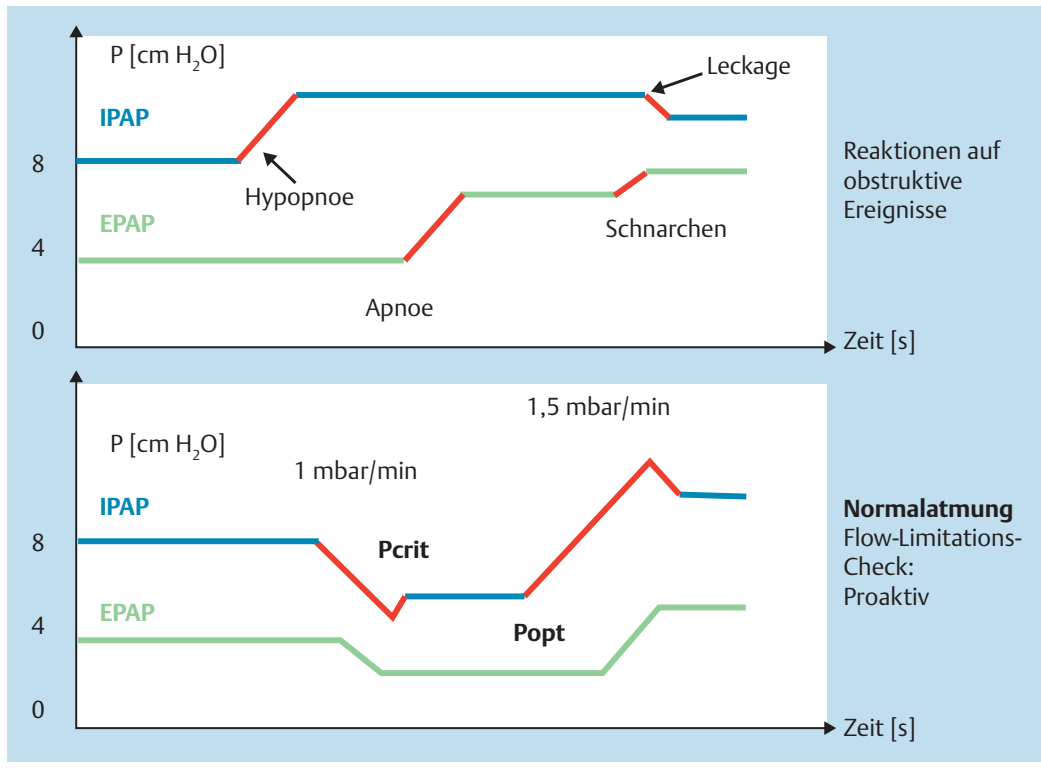

Abb.4 Auto-BIPAP: a Bei Erkennung obstruktiver Ereignisse wie Apnoen und Schnarchen wird der EPAP erhöht. Wenn Hypopnoen erfasst werden, wird der IPAP erhöht. Bei Überschreiten einer Leckage-Schwelle wird der IPAP abgesenkt. b Bei länger dauernder Normalatmung > 10 Minuten wird der IPAP langsam abgesenkt, bis eine Flusslimitation zu erkennen ist (Pcrit). Bei Flusslimitation wird IPAP bis zur Normalisierung erhöht (Popt).

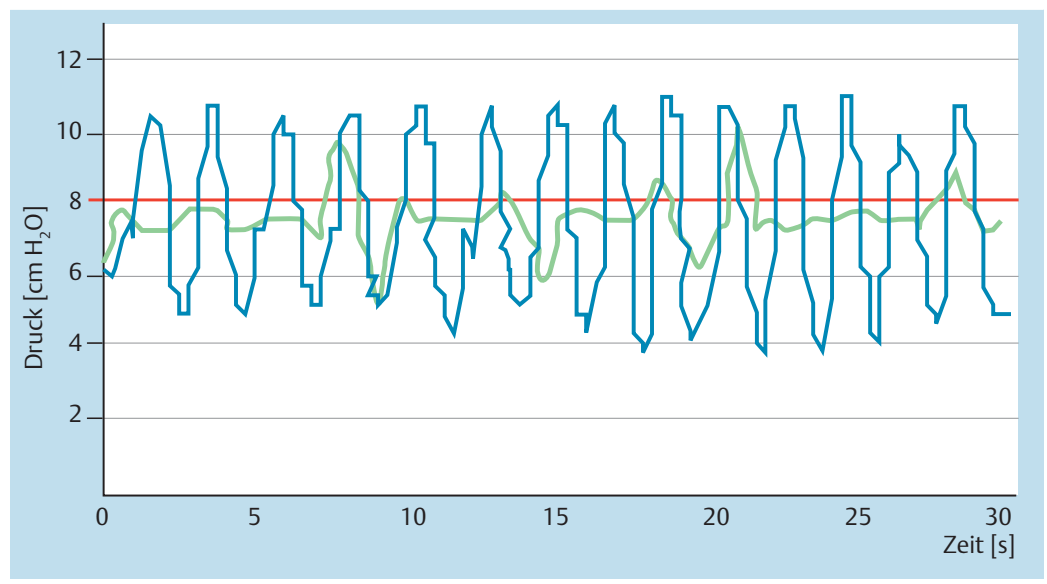

Abb.5 Atemzyklus gesteuerte Druckmodulation (Flexline-Modus). Dynamische Druckkurve mit inspiratorischer Druckanhebung und exspiratorischer Druckabsenkung. Eingestellter Druck $8 \mathrm{~cm}$ $\mathrm{H}_{2} \mathrm{O}$, Flexstufe 3 (Rote Linie). Druckverlauf bei geringer Atemfrequenz von 10/min (grüne Kurve) Druckverlauf bei hoher Atemfrequenz von 25/min (blaue Kurve). Modifiziert nach Nilius et al. [24].

Probanden wurde das Verfahren getestet, wobei unterschiedliche Atemfrequenzen vorgegeben wurden [24].

Bei einer Atemfrequenz von 25 und einem CPAPWert von $8 \mathrm{~cm} \mathrm{H}_{2} \mathrm{O}$ lag die Amplitude (Differenz zwischen maximaler Inspiration und maximaler

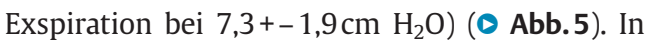
der klinischen Testung wurde das konventionelle Verfahren mit CPAP mit der in- und exspiratorischen Druckvariation verglichen. Es zeigte sich, dass die sinusoidale Druckapplikation weder zur Hyperventilation mit Zunahme zentraler Apnoen führte noch Auswirkungen auf die Schlafqualität nach sich zog. Ein Vorteil gegenüber dem konventionellen CPAP-Verfahren konnte nicht objekti-

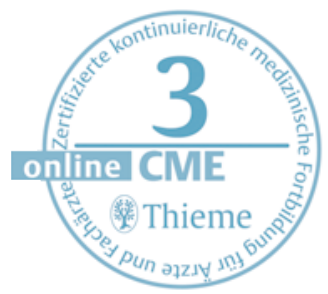


Abb.6 Schema der inspiratorischen Druckunterstützung: Im Rahmen der CPAP-Therapie kann die C-Flex ${ }^{\circledR}$-Technologie zugeschaltet werden, bei C-Flex ${ }^{\circledR}$ wird neben der exspiratorischen Druckentlastung der Druck zusätzlich während der Inspiration erhöht. Bei der APAP-Therapie kann ebenfalls der C-Flex ${ }^{\circledR}$ Modus aktiviert werden, bei A-Flex ${ }^{\circledR}$ wird zusätzlich zu C-Flex ${ }^{\circledR}$ eine inspiratorische Druckunterstützung gewählt.

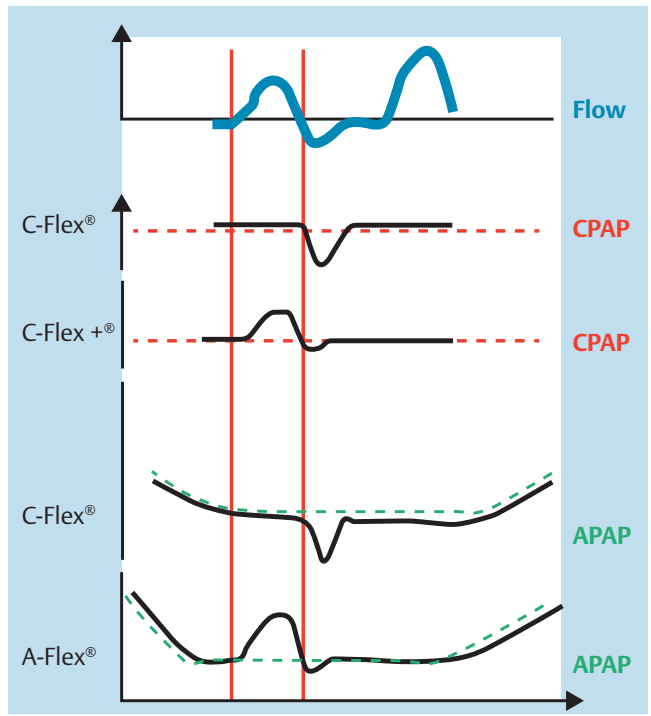

viert werden. Wie bei allen anderen Druckmodifikationen bleibt es dem erfahrenen Somnologen überlassen, welche Therapieform bei Intoleranz zum Einsatz kommt.

Einen dem Flexline ${ }^{\circledR}$-Druckverfahren ähnlichen Druckverlauf findet man bei der A-Flex ${ }^{\circledR}$-Methode $(\bullet$ Abb.6)

Bei jeweils 30 Patienten wurde APAP mit C-Flex ${ }^{\circledR}$ und A-Flex verglichen [25]. Unter APAP mit CFlex konnte gegenüber APAP allein eine signifikante Zunahme der Adhärenz mit 5,2+-1,8 versus 4,0+-1,7 Stunden beobachtet werden. APAP mit A-Flex erbrachte keinen zusätzlichen Vorteil.

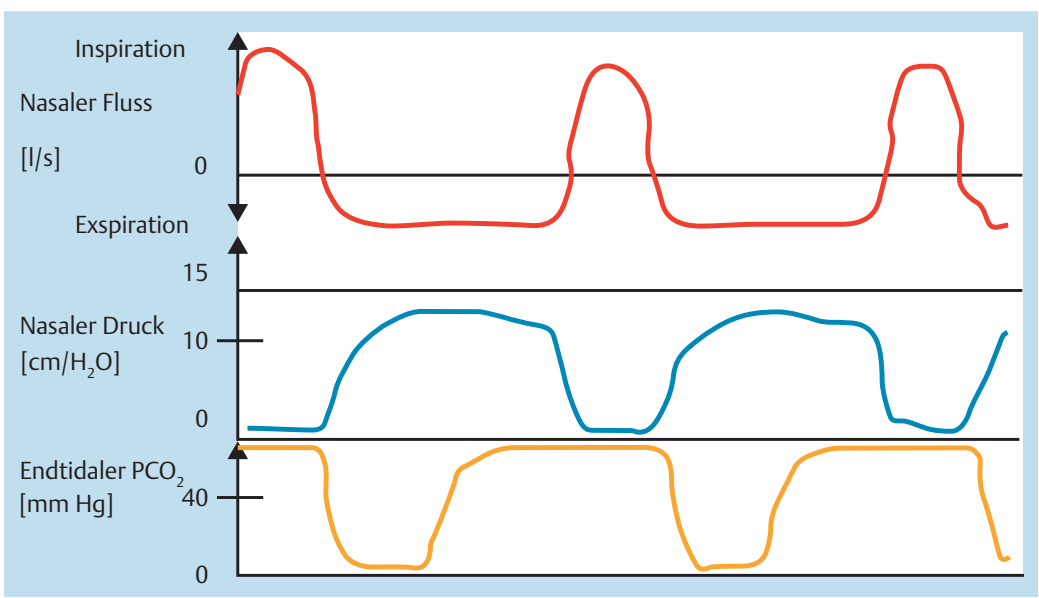

Abb.7 Fluss- und Druckverlauf unter EPAP-Atmung (EPAP = exspiratorisch positiver Atemwegsdruck). Druckanstieg in der Exspiration auf $13 \mathrm{~cm} \mathrm{H}_{2} \mathrm{O}$, Abfall auf $0 \mathrm{~cm} \mathrm{H}_{2} \mathrm{O}$ während Inspiration. Der endtidale $\mathrm{PCO}_{2}$ steigt deutlich über $40 \mathrm{~mm} \mathrm{Hg}$. Modifiziert nach Braga et al [29].
Verfahren mit exspiratorisch erhöhtem positiven Atemwegsdruck

\section{$\boldsymbol{\nabla}$}

EPAP (Exspiratorisch positiver Atemwegsdruck)

Um die Therapie mit positivem Atemwegsdruck bei OSA zu vereinfachen, wurde überprüft, ob eine effektive Therapie durch alleinige Erhöhung des exspiratorischen Druckes mit nasalen Ventilen, die sich während der Einatmung öffnen, aber während der Ausatmung einen hohen Widerstand (etwa $80 \mathrm{~cm} \mathrm{H}_{2} \mathrm{O} / \mathrm{L} / \mathrm{sec}$ bei einer Flussrate von $100 \mathrm{~mL} / \mathrm{sec}$ ) bieten, möglich ist ( $\bullet$ Abb.7). In einer Pilotstudie konnte durch in die Nasenöffnungen eingefügten Ventile der AHI von 24,8 auf 14,2 /h reduziert werden (Provent ${ }^{\circledR}$ Sleep Apnea Therapy, Ventus Medical). Der prozentuale Anteil von Schnarchen verringerte sich von 26,9 auf $9,4 \%$ des Nachtschlafs [26].

Es handelt sich um selbstklebende Ventile, die nur einmal verwendet werden sollen. Im Vergleich zu einer randomisierten Vergleichsgruppe mit Schein-CPAP fiel der AHI von $16 / \mathrm{h}$ auf $8 / \mathrm{h}$ in der Behandlungsgruppe mit EPAP: die Tagesschläfrigkeit, gemessen mit dem Score der Epworth Sleepiness Scale (ESS), fiel von mehr als 13 signifikant auf unter 8 [27]. Auch die Langzeitakzeptanz nach 12 Monaten bei denjenigen Patienten, die nach 3 Monaten profitierten, war zufriedenstellend [28]. Folgende Erklärungen werden für die Wirksamkeit der Methode angeführt: Da vor dem Beginn der Einatmung ein positiver Atemwegsdruck herrscht, ist die Kollapsneigung zu Beginn der Inspiration verringert. Zudem erzeugt der erhöhte Druck am Ende der Ausatmung ein erhöhtes Lungenvolumen, sodass die Zugspannung des oberen Atemwegs zunimmt und dieser damit stabilisiert wird [29].

\section{Adaptive Verfahren \\ $\nabla$}

\section{SPAP (Adaptive Servoventilation, ASV)}

Zentrale Schlafapnoe (CSA) und Cheyne-Stokes Atmung (CSR) sind häufig nur unzureichend durch CPAP zu beseitigen. Lediglich etwa 50\% aller Patienten sprechen auf diese Therapie an. Auch andere Therapieverfahren wie BIPAP-ST führten nicht zu dem erwarteten Erfolg, da eine fixe Druckunterstützung die Atmung nicht stabilisiert.

Bei der adaptiven Servoventilation wird ein fixer Ausatmungsdruck (EPAP) gewählt, der auch auf Automatik mit variablem Druck (Auto-EPAP) eingestellt werden kann. Es hat sich bewährt, dass der EPAP in der ersten Einstellungsnacht durch Titration bestimmt wird. Der Ausatmungsdruck (EPAP) dient zum Offenhalten der oberen Atemwege ( $\bullet$ Abb. 8). 
Zu Beginn einer Einstellung auf ASV werden zuerst die Atmungscharakteristika des jeweiligen Patienten erfasst und analysiert. Damit gelingt es, ein individuelles Flussverhalten zu definieren.

Bei der CSR variiert das Tidalvolumen mit einem Crescendo- und einem Decrescendo-Muster. Durch die antizyklisch modulierte Ventilation erfolgt eine variable inspiratorische Druckunterstützung (IPAP): Wenn ein erhöhter Fluss gemessen wird, wird der IPAP reduziert, bei geringem Fluss wird der IPAP erhöht ( $\bullet$ Abb.9, $\bullet$ Abb. 10). Als Zielgröße dient der über ein gleitendes Fenster von 3-4 min jeweils ermittelte Atemfluss.

Die modernen Geräte synchronisieren die Druckunterstützung, indem die letzte durchschnittliche Atemfrequenz, das zeitliche Verhältnis von Inund Exspiration (ti/te-Verhältnis), die Länge der Apnoen, die Richtung, Größe und Änderung des Flows und die Atemfrequenz kontinuierlich erfasst werden.

Eine Metaanalyse von 14 Studien bei Patienten mit Herzinsuffizienz und CSA/CSR verglich CPAP mit ASV. Gezeigt wurde, dass ASV bzgl. AHI-Reduktion und LVEF-Zunahme effektiver war [30]. In einer randomisierten Multicenterstudie nutzten die Patienten die ASV-Therapie im Vergleich zu CPAP signifikant länger (5,2 $\pm 0,9$ vs $4,1 \pm 1,1 \mathrm{~h} /$ Nacht) [31].

In einer weiteren Studie wurde bei 70 Patienten mit Herzinsuffizienz mit sowohl zentraler als auch obstruktiver Schlafapnoe CPAP mit ASV verglichen [32]. Das ASV-Verfahren war bei einer Kontrolluntersuchung nach einem Jahr signifikant effektiver bezüglich der Reduktion des CSAIndex sowie des BNP-Spiegels.

Unklar ist bis jetzt allerdings, ob sich durch die PAP-Therapie eine Verbesserung der Prognose erzielen lässt [33].

Die adaptive Servoventilation (ASV) mit antizyklischer Ventilation ist die zur Zeit effektivste Therapie des zentralen Schlafapnoesyndroms.

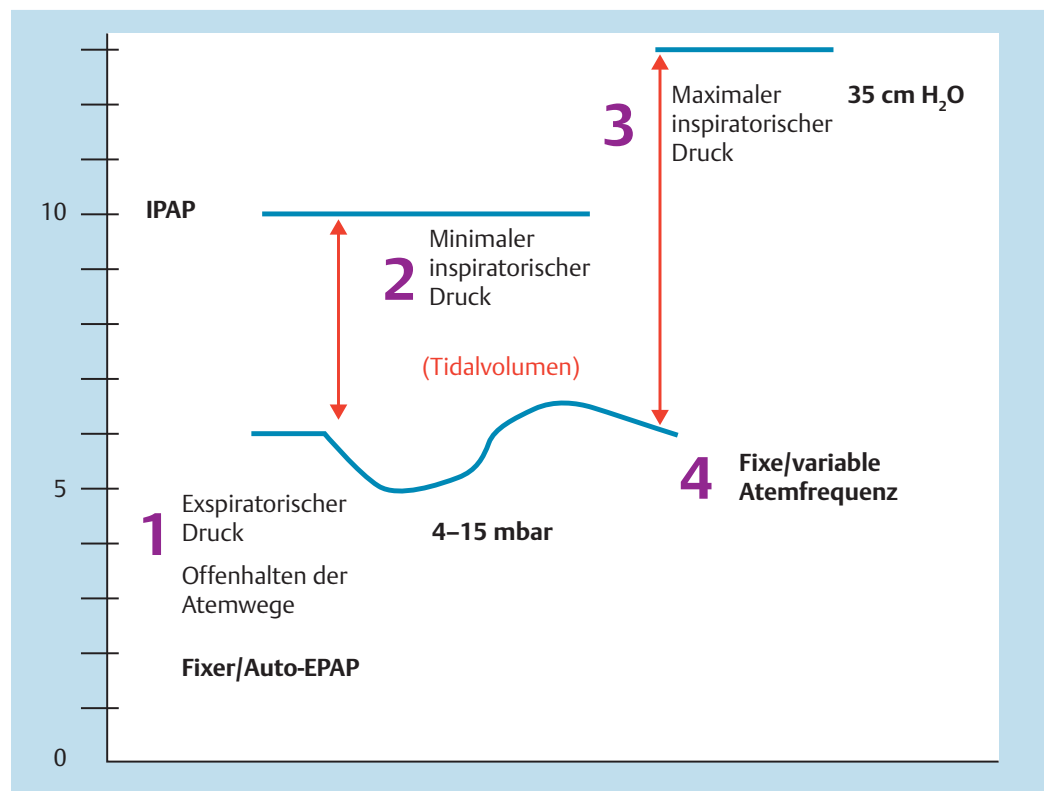

Abb. 8 S-PAP = Servo-PAP = ASV = Adadaptive Servo-Ventilation. Vorgehen bei der Druckeinstellung: 1 Festlegung des EPAP oder auto EPAP. 2 Festlegen des minimalen IPAP. 3 Festlegen des maximalen IPAP. 4 Fixe Backup-Atemfrequenz (oder automatisch). Durch den Gerätealgorithmus erfolgt die Synchronisation mit der Atmung des Patienten anhand des initialen Flows, der letzten durchschnittlichen Atemfrequenz, des ti/te-Verhältnisses, der Länge der Apnoen, der Richtung, Größe und Änderung des Flows.

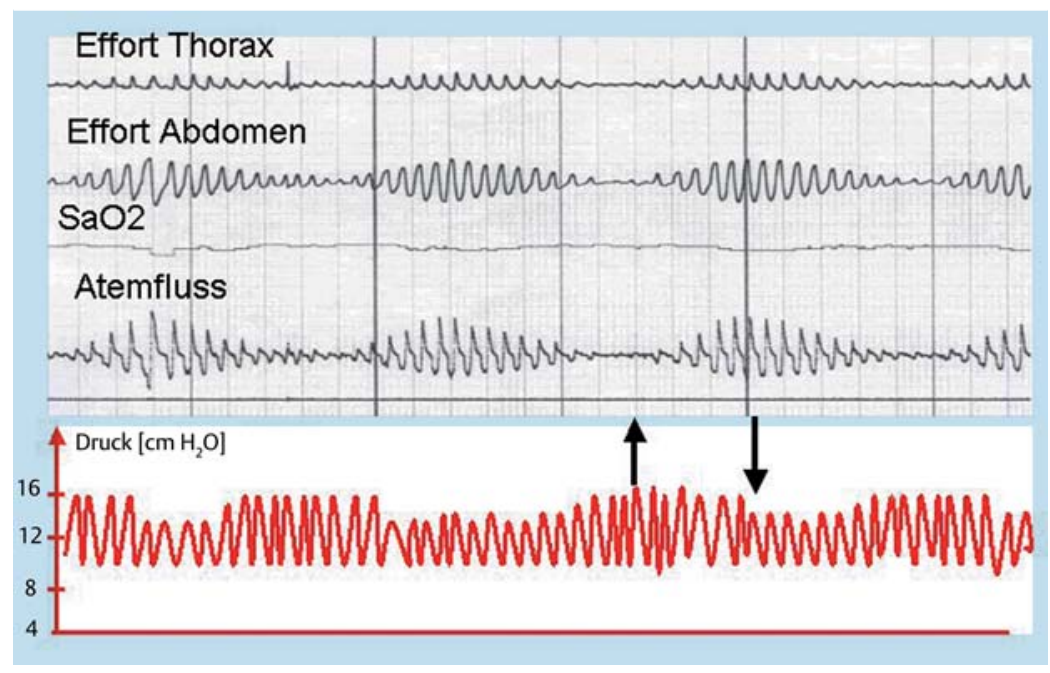

Abb.9 Schema der Druckunterstützung unter adaptiver Servoventilation (rote Kurve) bei Cheyne-Stokes-Atmung (schwarze Kurve). Die Cheyne-Stokes-Atmung ist durch ein zyklisches Atemmuster mit Crescendo- und Decrescendo-Atmung charakterisiert. In den Phasen der Hyperventilation wird die Druckunterstützung reduziert, während der Phasen mit Hypoventilation wird die Druckunterstützung erhöht.

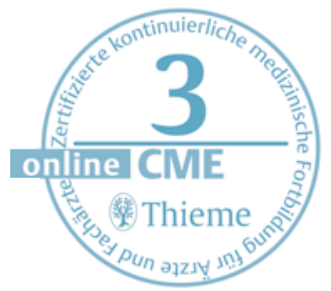




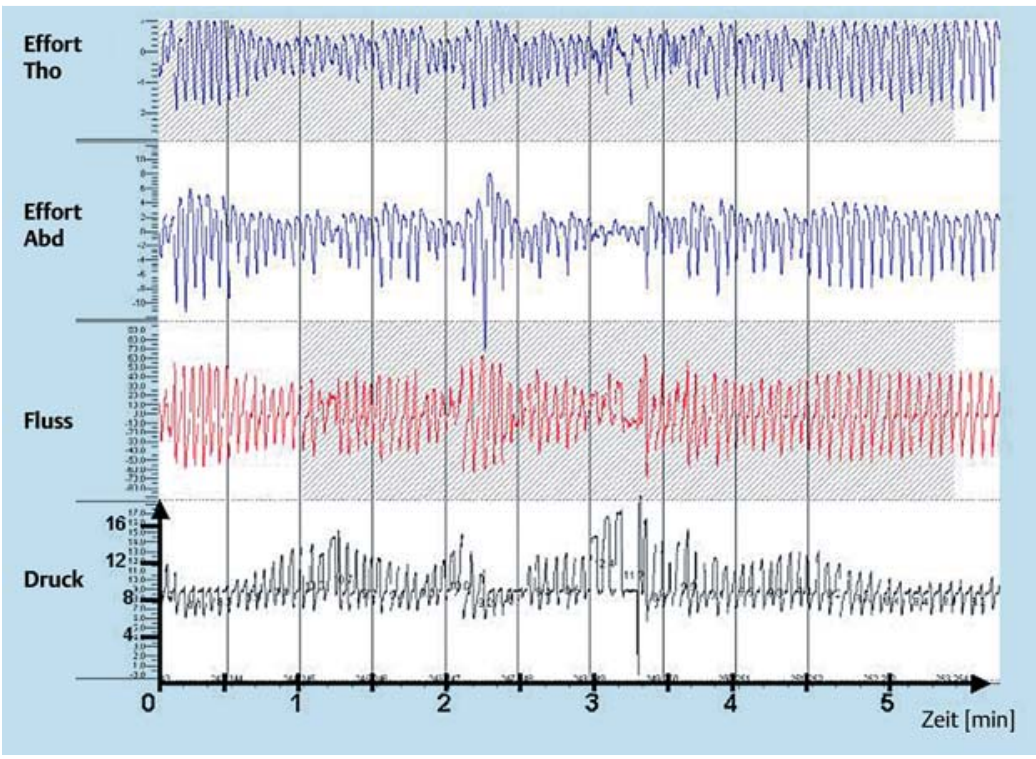

Abb.10 Polygrafie im frühen Verlauf einer adaptiven Servoventilation (ASV). Die Druckunterstützung bis zu 17 mbar (siehe unterste Kurve) während der Phasen mit Hypoventilation verhindert eine relevante Verminderung des Atemflusses. Die Flussamplituden zeigen aber noch ein deutliches Crescendo-und Decrescendo-Muster. Nach wenigen Stunden entwickelt sich unter ASV ein regelmäßiges Flussprofil.

\section{Schlussfolgerungen}

Die Therapie mit CPAP ist in den meisten Fällen bei obstruktiver Schlafapnoe erfolgreich. Bei nicht ausreichender Adhärenz bieten CPAP-Variationen die Möglichkeit der Differenzialtherapie. Objektive, relevante Verbesserungen der Adhärenz durch verschiedene Druckentlastungsverfahren konnte in Untergruppen von OSA nachgewiesen

\section{Literatur}

1 Galetke W, Puzzo L, Priegnitz C et al. Long-term therapy with continuous positive airway pressure in obstructive sleep apnea: adherence,side effects and predictors of withdrawal - a 'real-life' study. Respiration 2011; 82: $155-161$

2 Weaver TE, Maislin G, Dinges DF et al. Relationship between hours of CPAP use and achieving normal levels of sleepiness and daily functioning. Sleep 2007; 30: 711 - 9

3 Lewis KE, Seale L, Bartle IE et al. Early predictors of CPAP use for the treatment of obstructive sleep apnea. Sleep 2004; 27: 134-138

4 Kushida CA, Littner MR, Hirshkowitz M. American Academy of Sleep Medicine et al. Practice parameters for the use of continuous and bilevel positive airway pressure devices to treat adult patients with sleep-related breathing disorders. Sleep 2006; 29: 375-380

5 Parke RL, Eccleston ML, McGuinness SP. The effects of flow on airway pressure during nasal high-flow oxygen therapy. Respir Care 2011; 56: 1151-1155

6 McGinley BM, Patil SP, Kirkness JP et al. A nasal cannula can be used to treat obstructive sleep apnea. Am J Respir Crit Care Med 2007; 176: 194-200

7 Nilius G, Wessendorf T, Maurer J et al. Predictors for treating obstructive sleep apnea with an open nasal cannula system (transnasal insufflation). Chest 2010; 137: 521 528

8 Ruhle KH, Domanski U, Nilius G. Obstructive pressure peak: a new method for differentiation of obstructive and central apneas under auto-CPAP therapy. Sleep Breath 2013; 17: $111-115$

9 Randerath WJ, Schraeder O, Galetke W et al. Autoadjusting CPAP therapy based on impedance efficacy, compliance and acceptance. Am J Respir Crit Care Med 2001; 163: $652-657$

10 Massie CA, McArdle N, Hart RW et al. Comparison between automatic and fixed positive airway pressure therapy in the home. Am J Respir Crit Care Med 2003; 167: $20-23$

11 Sériès F, Marc I. Importance of sleep stage- and body position-dependence of sleep apnoea in determining benefits to auto-CPAP therapy. Eur Respir J 2001; 18: 170 175

12 Smith I, Lasserson TJ. Pressure modification for improving usage of continuous positive airway pressure machines in adults with obstructive sleep apnoea. Cochrane Database Syst Rev 2009: CD003531

13 Ayappa I, Norman RG, Whiting $D$ et al. Irregular respiration as a marker of wakefulness during titration of CPAP. Sleep 2009; 32: 99 - 104

14 Rühle KH, Karweina D, Domanski U et al. Clinical views on biosignal processing of ventilation in sleep medicine. Pneumologie 2011; 65: 89-93

15 Dungan GC2nd, Marshall NS, Hoyos CM et al. A randomized crossover trial of the effect of a novel method of pressure control (SensAwake)in automatic continuous positive airway pressure therapy to treat sleep disordered breathing. J Clin Sleep Med 2011; 7: 261 -267

16 Sanders MH, Kern N. Obstructive sleep apnea treated by independently adjusted inspiratory and expiratory positive airway pressures via nasal mask. Physiologic and clinical implications. Chest 1990; 98: $317-324$

17 Sanders MH, Montserrat JM, Farré R et al. Positive pressure therapy: a perspective on evidence-based outcomes and methods of application. Proc Am Thorac Soc 2008; 5: $161-172$

18 Resta O, Guido P, Picca V et al. Prescription of nCPAP and nBIPAP in obstructive sleep apnoea syndrome: Italian experience in 105 subjects. A prospective two centre study. Respir Med 1998; 92: 820-827

19 Schwartz SW, Rosas J, lannacone MR et al. Correlates of a prescription for Bilevel positive airway pressure for treatment of obstructive sleep apnea among veterans. J Clin Sleep Med 2013; 9: $327-335$ 
20 Rühle KH, Domanski U, Happel A et al. Analysis of expiratory pressure reduction (C-Flex method) during CPAP therapy]. Pneumologie 2007; 61: 86 - 89

21 Nilius G, Happel A, Domanski U et al. Pressure-relief continuous positive airway pressure vs constant continuous positive airway pressure: a comparison of efficacy and compliance. Chest 2006; 130: 1018-1024

22 Ballard RD, Gay PC, Strollo PJ. Interventions to improve compliance in sleep apnea patients previously non-compliant with continuous positive airway pressure. J Clin Sleep Med 2007; 3: 706-712

23 Powell ED, Gay PC, Ojile JM et al. A pilot study assessing adherence to auto-bilevel following a poor initial encounter with CPAP. J Clin Sleep Med 2012; 8: 43-47

24 Nilius G, Burian S, Franke KJ et al. Effects of a breath-triggered modulation of positive airway pressure ventilation on the AHI from patients with obstructive sleep apnoea. Pneumologie 2009; 63: 433-438

25 Chihara Y, Tsuboi T, Hitomi T et al. Flexible positive airway pressure improves treatment adherence compared with auto-adjusting PAP. Sleep 2013; 36: 229-236

26 Colrain IM, Brooks S, Black J. A pilot evaluation of a nasal expiratory resistance device for the treatment of obstructive sleep apnea. J Clin Sleep Med 2008; 4: 426 433

27 Berry RB, Kryger MH, Massie CA. A novel nasal expiratory positive airway pressure (EPAP) device for the treatment of obstructive sleep apnea: a randomized controlled trial. Sleep 2011; 34: 479-485
28 Kryger MH, Berry RB, Massie CA. Long-term use of a nasal expiratory positive airway pressure (EPAP) device as a treatment for obstructive sleep apnea (OSA). J Clin Sleep Med 2011; 7: 449-453

29 Braga $C W$, Chen $Q$, Burschtin $O E$ et al. Changes in lung volume and upper airway using MRI during application of nasal expiratory positive airway pressure in patients with sleep-disordered breathing. J Appl Physiol 2011; 111: $1400-1409$

30 Sharma BK, Bakker JP, McSharry DG et al. Adaptive servoventilation for treatment of sleep-disordered breathing in heart failure: a systematic review and meta-analysis. Chest 2012; 142: 1211 - 1221

31 Kasai T, Usui Y, Yoshioka $T$ et al. Effect of flow-triggered adaptive servo-ventilation compared with continuous positive airway pressure in patients with chronic heart failure with coexisting obstructive sleep apnea and Cheyne-Stokes respiration. Circ Heart Fail 2010; 3: $140-148$

32 Randerath WJ, Nothofer G, Priegnitz C et al. Long-term auto-servoventilation or constant positive pressure in heart failure and coexisting central with obstructive sleep apnea. Chest 2012; 142: 440 - 447

33 Zeller J, Hetzenecker A, Arzt M. Sleep-disordered breathing in patients with chronic heart failure: epiphenomenon or bidirectional relationship. Pneumologie 2013; 67: $150-156$ 


\section{CME-Fragen X-PAP-Therapie bei schlaf- bezogenen Atmungsstörungen}

1 Welche Aussage zur automatischen Drucksteuerung

(APAP) bei obstruktiver Schlafapnoe ist falsch?

A Bei erniedrigter Sauerstoffsättigung wird bei der APAP-Therapie automatisch der Druck erhöht.

B Bei erniedrigtem Atemfluss zum Beispiel unter 30\% der durchschnittlichen Flussrate wird der Druck erhöht.

C Die Detektion von Schnarchen kann zu einer Druckerhöhung führen.

D Flattening, d.h. die Abflachung des Flusssignals, ist ein Indiz für eine Widerstandserhöhung in den oberen Luftwegen und kann zu einer Druckerhöhung führen.

E Die forcierte Oszillations-Technik dient zur Messung des Widerstandes in den oberen Atemwegen und kann zur Steuerung von APAP verwendet werden.

A APAP empfiehlt sich, wenn ein geringer Druck bei der Titration ermittelt wurde.

B Wenn der Patient über ausgetrocknete Schleimhäute klagt, sollte primär ein APAP-Gerät verordnet werden.

C Wenn bei der CPAP-Titration ein hoher Druckbedarf ermittelt wurde, ist bei Verordnung eines APAP-Gerätes eine im Vergleich zu CPAP höhere Nutzungszeit zu erwarten.

D Die meisten Patienten bevorzugen bei Vergleich von CPAP und APAP die CPAP-Therapie.

E Bei ausgeprägter Lageabhängigkeit der Obstruktion ist APAP nicht indiziert.

Welche Aussage ist im Zusammenhang mit der Detektion von Wachphasen unter APAP richtig?

A In dem APAP Algorithmus werden die Ergebnisse des Elektro-Okulogramms (EOG) berücksichtigt.

B Ein unregelmäßiges Flussmuster mit Amplitudenund Frequenzschwankungen kann als Hinweis für Wachphasen und als Steuersignal für ein APAP-Gerät verwendet werden.

C Bei Registrierung von wiederholten Apnoen in der Wachphase wird der Druck durch das APAP-Gerät schnell abgesenkt.

D Vor allem während der Wachphasen ist ein hoher Atemwegsdruck erforderlich.

E Wachphasen können nur mittels Polysomnografie erfasst werden.
4 Welche Aussage zur Druckentlastung (pressure relief) unter CPAP ist richtig?

A Pressure Relief ist eine Methode zur Erkennung von Wachphasen.

B Von einer Druckentlastung profitieren die Patienten vor allem während der Inspiration.

C Die Druckentlastung wird vor allem während der Exspiration wirksam.

D Unter CPAP mit Druckentlastung wird der Druck während der gesamten Exspiration bis zur nächsten Inspiration abgesenkt.

E Die Druckabsenkung während der Exspiration wird durch den inspiratorischen Fluss gesteuert.

\section{Welche}

A Die Therapie mit ASV ist vor allem bei obstruktiver Schlafapnoe indiziert.

B ASV reduziert die Hyperkapnie bei chronisch obstruktiver Lungenerkrankung (COPD).

C Adaptive Servoventilation verhindert die Obstruktion bei Asthma bronchiale.

D Adaptive Servoventilation stellt die optimale Möglichkeit zur assistierten Beatmung bei OverlapSyndrom dar.

E Zentrale Schlafapnoen (CSA) und Cheyne-StokesAtmung (CSR) sind häufig nur durch ASV zu beseitigen.

Welche Aussage zur Therapie mit exspiratorisch positivem Atemwegsdruck (EPAP) ist richtig?

A EPAP führt zu einer Erhöhung des Apnoe/HypopnoeIndex.

B Die Therapie mit EPAP stellt ein aufwändiges apparatives Verfahren dar.

C Die exspiratorische Druckerhöhung wird durch Ventile in beiden Nasenöffnungen mit geringem Widerstand während der Ausatmung erzeugt.

D Einer der Wirkmechanismen von EPAP beruht auf der Erhöhung des endexspiratorischen Lungenvolumens und Stabilisierung der oberen Atemwege.

E Die Therapie mit exspiratorisch positivem Atemwegsdruck wirkt nur bei primärem Schnarchen. 
7 Welche Aussage zur adaptiven Servoventilation (ASV) ist falsch?

A Der Ausatmungsdruck (EPAP) dient zum Offenhalten der oberen Atemwege.

B Zu Beginn einer Einstellung auf ASV prüft das Gerät zuerst den Atemfluss des Patienten zur Charakterisierung des Atemmusters.

C Die zusätzliche Druckunterstützung durch ASV erfolgt jeweils bei gesteigerter Ventilation.

D Wenn ein erhöhter Atemfluss des Patienten gemessen wird, reduziert das Gerät die Druckunterstützung, bei geringem Atemfluss wird die Druckunterstützung erhöht.

E Auch bei längerer Anwendung scheint das ASV-Verfahren im Vergleich zu CPAP effektiver bezüglich der Reduktion des CSA-Index sowie des BNP-Spiegels zu sein.

Welche Aussage bezüglich Therapieadhärenz ist falsch?

A Die häusliche CPAP-Therapiedauer pro Nacht sollte in Abhängigkeit vom klinischen Ansprechen festgelegt werden.

B Eine Mindesttherapiedauer von 6 Stunden pro Nacht ist in jedem Fall erforderlich.

C Die Adhärenz hängt von der Beatmungsqualität ab.

D Eine ausreichende Adhärenz ist zu erwarten, wenn während der ersten Titrationsnacht mit CPAP keine relevanten Probleme berichtet werden.

E Selbst bei optimaler Betreuung nutzen nur etwa 75\% der OSA Patienten die XPAP-Therapie.
9 Welche Aussage bezüglich nasaler High-Flow-Therapie ist richtig?

A Mit transnasaler Insufflation wird supraglottisch bei einer Flussrate von 201/min ein Druck von etwa $6 \mathrm{~cm}$ $\mathrm{H}_{2} \mathrm{O}$ gemessen.

B Auch bei einem dünnen Schlauch von wenigen Millimetern reicht der vom Kompressor erzeugte Druck von 50 mbar aus, um einen Fluss von $301 /$ min zu erzeugen.

c Um die Abkühlung der Nasenschleimhäute durch die hohe Flussrate zu verhindern, wird die Atemluft durch eine Schlauchheizung auf $37^{\circ} \mathrm{C}$ angewärmt.

D Die Luftfeuchtigkeit bei der nasalen High-FlowTherapie sollte mindestens $40 \%$ betragen.

E Mittels transnasaler Insufflation kann in den oberen Atemwegen ein Druck von etwa 2 bis $3 \mathrm{~cm} \mathrm{H}_{2} \mathrm{O}$ erzeugt werden.

Welche Aussage bezüglich des klinischen Effekts einer nasalen High-Flow-Therapie ist richtig?

A Von einer nasalen High-Flow-Therapie profitieren am meisten OSA-Patienten mit überwiegend Hypopnoen.

B Bei Patienten mit überwiegend Schnarchen ist eine nasale High-Flow-Therapie nicht indiziert.

c Von einer nasalen High-Flow-Therapie profitieren am ehesten OSA-Patienten mit einem hohen ApnoeIndex.

D High-Flow-Therapie stellt eine Alternative zur adaptiven Servoventilation dar.

E Die nasale High-Flow-Therapie wird hauptsächlich über eine nasale Maske appliziert. 